\title{
Diagnóstico espaço-temporal dos focos de calor no Brasil
}

\author{
Diagnosis spatiotemporal of fire foci in Brazil \\ Análisis espacio-temporal de focus de calor em Brasil
}

Recebido: 28/05/2021 | Revisado: 06/06/2021 | Aceito: 11/06/2021 | Publicado: 25/06/2021

\author{
Carla Taciane dos Santos \\ ORCID: https://orcid.org/0000-0002-6426-9346 \\ Universidade Federal de Alagoas, Brasil \\ E-mail: carlataciane01@gmail.com \\ Washington Luiz Félix Correia Filho \\ ORCID: https://orcid.org/0000-0002-4029-4491 \\ Universidade Federal de Alagoas, Brasil \\ E-mail: wlfcfm@gmail.com \\ José Francisco de Oliveira Júnior \\ ORCID: https://orcid.org/0000-0002-6131-7605 \\ Universidade Federal de Alagoas, Brasil \\ E-mail: jose.junior@icat.ufal.br \\ Dimas de Barros Santiago \\ ORCID: https://orcid.org/0000-0001-7118-8467 \\ Universidade Federal de Campina Grande, Brasil \\ E-mail: dimas.barros91@gmail.com \\ Bárbara Alves Batista \\ ORCID: https://orcid.org/0000-0002-0034-0991 \\ Universidade Federal de Alagoas, Brasil \\ E-mail: barbara.alba18@gmail.com
}

\begin{abstract}
Resumo
O surgimento de incêndios florestais pode ser de origem antrópica ou natural, ambas causam grandes prejuízos socioeconômico e ambiental, e em boa parte dessas ocorrências são resultantes da ocorrência de Focos de Calor (FC). Nos últimos anos, o Brasil tem sofrido com o aumento significativo de FC, ao qual resultaram em grandes incêndios. Desta maneira, o objetivo do presente estudo foi diagnosticar o comportamento espaço-temporal dos FC no Brasil entre 1999 e 2020, baseados nos dados de dados BDQueimadas do CPTEC/INPE. Para a manipulação e o processamento dos dados, utilizou-se o software de ambiente R versão 3.4-1. Após o armazenamento dos dados, calculou-se os registros totais, médias anual e mensal, e a composição dos anos mais significativos, neste caso, os anos de 2015, 2017, 2019 e 2020. Os resultados apontaram que os maiores acumulados totais e médios anuais variaram entre 10-50 mil FC e 0,5-1,5 mil FC, concentrados na região centro-norte do Brasil, principalmente nos estados do Maranhão, Pará e Tocantins. Este padrão de alto registros de FC está relacionado ao desmatamento e expansão agrícola nessas regiões. Em escala mensal, as maiores ocorrências de FC ocorrem entre os meses de agosto e novembro, com valores de 0,20-0,45 mil FC, devido ao período de estiagem. Verificou-se que nos últimos anos, o El Niño-Oscilação Sul influenciou a incidência dos FC através da persistência de longos períodos de estiagem, que resultaram em escassez de chuvas e grandes incêndios verificados em 2020 no bioma Pantanal.
\end{abstract}

Palavras-chave: Focos de calor; Desmatamento; Agricultura; MATOPIBA.

\begin{abstract}
The emergence of forest fires may be of anthropic or natural origin, both of which cause significant socio-economic and environmental damage, and in the good part of these occurrences are resulting from Fire Foci (FF) occurrence. In recent years, Brazil has suffered from a significant increase in FF, resulting in large fires. In this way, the objective of the present study was to diagnose the spatiotemporal FF behavior in Brazil between 1999 à 2020, based on the BDQueimadas data of the CPTEC/INPE. The handling and processing of the data used the $\mathrm{R}$ version 3.4-1 environment software. After the data storage, it calculated the total, annual, and monthly records. And the composition of the most significant years, in this case, the years 2015, 2017, 2019, and 2020. The results pointed out that the most significant total and average annual accumulations ranged between 10-50 thousand FF and 0.5-1.5 thousand FF, respectively, concentrated in the central-northern region of Brazil, mainly in Maranhão, Pará, and the Tocantins. This pattern of high FC records is related to deforestation and agricultural expansion in these regions. On a monthly scale, the most significant occurrences of FF occur between August and November, with 0.2-0.45 thousand FF, due to starvation. In recent years, the El Niño-Southern Oscillation influenced the annual FF performance through the persistence of long stretches of styling, which resulted in a shortage of rainfall and large fires verified in 2020 in the Pantanal biome.
\end{abstract}


Keywords: Fire foci; Deforestation; Agriculture; MATOPIBA.

\begin{abstract}
Resumen
La aparición de incendios forestales puede ser de origen antrópico o natural, los cuales causan importantes daños socioeconómicos y ambientales, y en buena parte de estas ocurrencias resultan de la ocurrencia de Focus de Calor (FC). En los últimos años, Brasil ha sufrido el aumento significativo del FC, al que han provocado grandes incendios. De esta manera, el objetivo del presente estudio fue diagnosticar el comportamiento espacio-temporal del FC en Brasil entre 1999 y 2020, basado en los datos BDQueimadas del CPTEC/INPE. Para el manejo y procesamiento de los datos, se utiliza el software de entorno R versión 3.4-1. Tras el almacenamiento de los datos, se calcularon los registros totales, anuales y mensuales, y la composición de los años más significativos, en este caso, los años 2015, 2017, 2019 y 2020. Los resultados señalaron que las acumulaciones anuales totales y promedio más grandes oscilaron entre 10-50 mil FC y 0,5-1,5 mil FC, concentradas en la región centro-norte del Brasil, principalmente en los estados de Maranhão, Pará y Tocantins. Este patrón de altos registros de FC está relacionado con la deforestación y la expansión agrícola en estas regiones. En una escala mensual, las ocurrencias más grandes de FC ocurren entre los meses de agosto y noviembre, con valores de 0,20-0,45 mil FC, debido al período de agitación. Se encontró que en los últimos años, la Oscilación del Niño-Sur influyó en la incidencia del FC a través de la persistencia de largos tramos de estilismo, lo que resultó en una escasez de lluvias y gran incendios verificados en 2020 en el bioma Pantanal.
\end{abstract}

Palabras clave: Focus de calor; Deforestación; Agricultura; MATOPIBA.

\title{
1. Introdução
}

O surgimento de incêndios florestais pode ser de origem antrópica ou natural, ambas causam grandes prejuízos para o meio ambiente, ao próprio homem e suas atividades econômicas (Caúla et al., 2015; Marinho et al, 2021; Oliveira-Júnior et al, 2021). No Brasil, é comum o uso do fogo para diversos fins, por exemplo, atividades de limpeza e manejo de pastagem, tanto na sua forma primitiva como em sistemas de produção intensivos, na eliminação de resíduos, pragas ou, ainda, na renovação de pastos e na limpeza de terrenos (Marinho et al., 2021). O uso das queimadas, embora seja economicamente viável em curto prazo pode trazer sérios danos a agricultura e pecuária, sobretudo em áreas de uso recorrente do fogo, pois causa empobrecimento do solo, ocasionando queda na produtividade agrícola (Assis et al., 2021; Oliveira et al., 2021).

Nesse cenário, o Brasil desponta como líder entre os países da América do Sul (AS) com a maior concentração de focos de calor (FC) nas regiões Centro-Oeste, Norte e Nordeste (Caúla et al., 2015). A Amazônia brasileira exibe um histórico de desmatamento desde a década de 1970, com o início da ocupação da região que incentivou um processo migratório e, assim um adensamento urbano (INPE, 2021). No entanto, nos últimos anos têm-se constatado um aumento significativo no número de $\mathrm{FC}$, possivelmente relacionados às alterações no padrão climático, em virtude da intensificação das mudanças climáticas e da atuação do modo da variabilidade climática El Niño-Oscilação Sul (ENOS) - (Caúla et al., 2015; Clemente et al., 2017; Urrutia-Jalabert et al., 2018). Outros fatores climáticos também intensificam os incêndios, por exemplo, períodos prolongados de estiagem, secas severas, velocidade do vento e o relevo, influenciam na propagação e determinam os seus efeitos devastadores (Xiong et al., 2020). Neste sentido, o uso de técnicas de Sensoriamento Remoto e Geoprocessamento tem sido eficazes no monitoramento do $\mathrm{FC}$, devido à sua agilidade e praticidade e, assim primordiais para a prevenção e controle de incêndio em áreas de vegetação (Caúla et al., 2015; Chuvieco et al., 2020). Portanto, o objetivo do presente estudo foi diagnosticar a variabilidade espaço-temporal dos FC no Brasil entre 1999 e 2020.

\section{Metodologia}

\section{1 Área de estudo}

O Brasil está localizado na porção centro-oriental da AS, com uma área de 8,515 milhões km² (IBGE, 2020), com aproximadamente 47\% do total (Figura 1). Atualmente, o Brasil está geopoliticamente dividido em 26 estados e um Distrito Federal; e subdividido em cinco regiões: i) Centro-Oeste, ii) Nordeste, iii) Norte, iv) Sudeste e v) Sul. De acordo com a classificação de Köppen descrito por Alvares et al. (2013), o Brasil apresenta clima predominantemente tropical (81\%), 
seguido dos climas subtropical úmido (14\%) e seco (5\%), além disso, apresenta uma estação chuvosa (verão) e outra seca (inverno) bem definidas.

Figura 1 - Delimitação do Brasil a partir da elevação (metros).

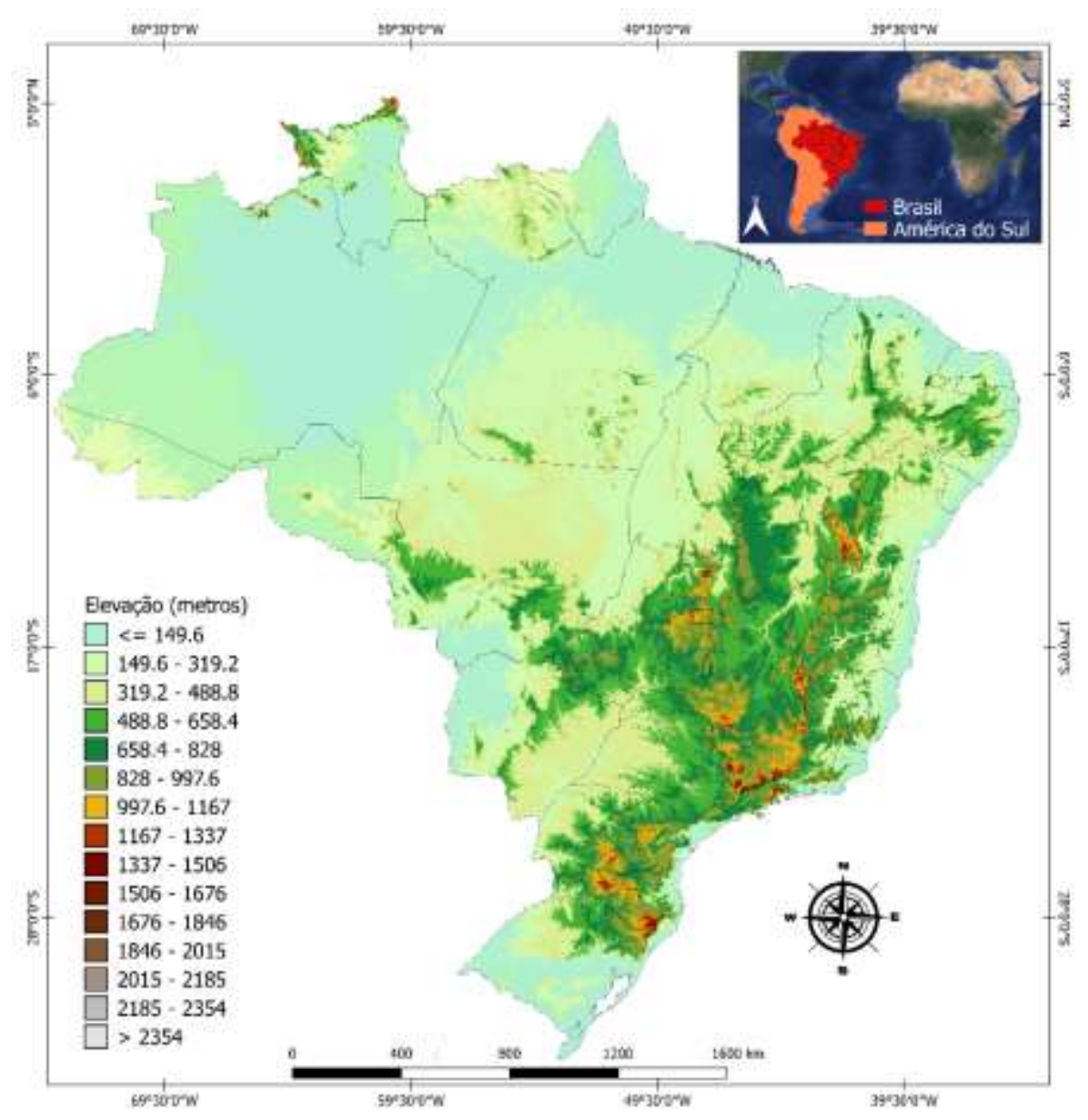

Fonte: Jarvis et al. (2008). Mapa elaborado pelos autores.

O relevo brasileiro é caraterizado por baixas e médias altitudes, com o predomínio de planaltos e depressões ao longo do país. O Brasil abrange seis tipos de cobertura vegetal: Floresta Amazônia, Mata Atlântica, Cerrado, Caatinga, Pantanal e Pampa. Apesar de essas vegetações sofrerem com o processo de desmatamento desde o período da colonização, elas ainda recobrem uma considerável parte do território nacional (Landau \& Moura, 2020).

\subsection{Dados}

Os dados de FC foram obtidos via Banco de Dados de Queimadas (BDQueimadas) no período de 1999 a 2020. No estudo foram utilizados os seguintes satélites ambientais: AQUA M-M, AQUA M-T, ATSR, GOES-8, GOES-10, GOES-12, GOES-13, NOAA-12, NOAA-12D, NOAA-12D, NOAA-12N, NOAA-14, NOAA-15, NOAA-15D, NOAA-16, NOAA-16N, NOAA-17, NOAA-18, NOAA-18D, NOAA-19, NOAA-19D, NPP-375,TRMM, TERRA M-T, e TERRA M-M que possuem sensores ópticos que operam na faixa termal-média de 3,7 $\mu \mathrm{m}$ a $4.1 \mu \mathrm{m}$, todos gerados e processados operacionalmente na Divisão de Satélites e Sistemas Ambientais (DSA) - (CPTEC/INPE, 2021). Atualmente, o CPTEC/INPE utiliza os satélites ambientais (Órbita Polar e Geoestacionário) em sua rede de monitoramento sobre a AS. Esses satélites realizam o imageamento orbital pelo continente Sul-americano ao longo do dia (CPTEC/INPE, 2021). Para a composição dos mapas de 
FC, todos os registros foram convertidos e armazenados em pontos de grade com resolução de $0,25^{\circ} \mathrm{x} 0,25^{\circ}$ (neste caso, se referem a $25 \mathrm{~km}$ x $25 \mathrm{~km}$ ), ao qual este procedimento foi realizado pelo software de ambiente R versão 3.6-1 (R Core Team Development, 2019).

\section{Resultados e Discussão}

Os resultados exibidos nesse tópico abordarão três aspectos: 1) total e a média anual, 2) anual, e 3) mensal. A Figura 2 mostra o total e a média anual de FC no Brasil. No Brasil, os acumulados totais de FC são altamente variáveis espacialmente ao longo do período de estudo, onde as maiores concentrações de FC estão situadas no Centro-Norte do Brasil, nos limites das regiões Norte, Nordeste e Centro-Oeste, entre os estados do Bahia, Maranhão, Mato Grosso, Pará, Piauí e Tocantins. As concentrações de registros totais (Figura 2a) variaram entre 5-42 mil FC, principalmente nas porções central do estado do Maranhão e sul do Tocantins. Outros estados se destacam como Alagoas (na porção leste) com valores entre 5-25 mil FC, enquanto Rondônia (na porção norte) e Mato Grosso do Sul (na porção leste) exibem altas concentrações (> 25 mil FC). No entanto, a maior parte do estado do Amazonas e Rio Grande do Sul exibem os menores registros de FC $(<0,5$ mil de FC).

Figura 2 - Distribuição espacial de FC no Brasil entre os anos de 1999 e 2020, relativos ao número total (esquerda) e média anual (direita) de ocorrências, respectivamente.
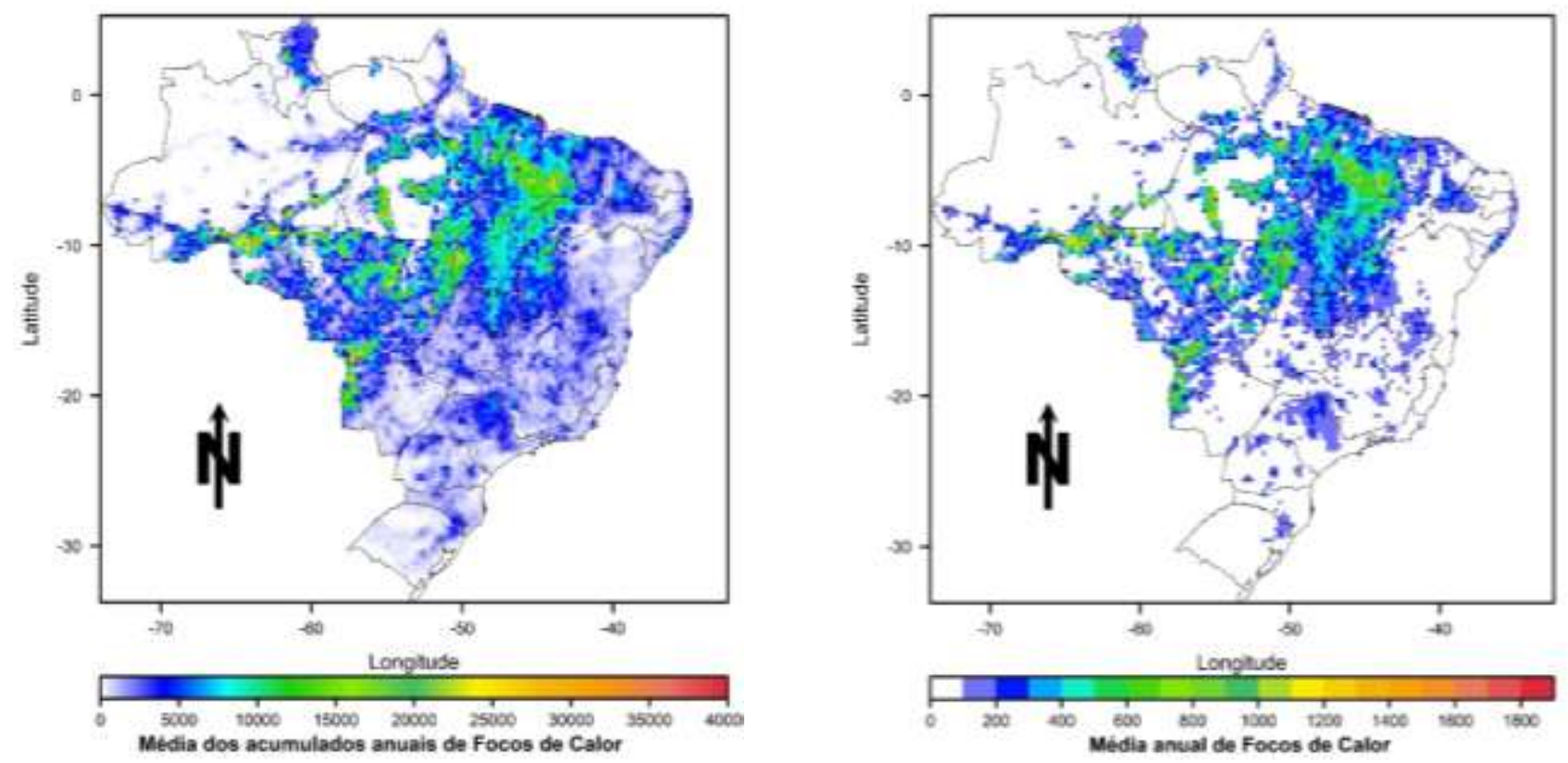

Fonte: BDQUEIMADAS (CPTEC/INPE, 2021). Mapa elaborado pelos autores.

Já o padrão médio anual (Figura 2b) exibe configuração similar ao total de FC, reiterando as regiões com as maiores concentrações situadas nos estados do Maranhão e Mato Grosso, com registros superiores entre 0,75-1,5 mil FC. Verifica-se também que o norte de Rondônia, leste do Mato Grosso do Sul, e no sul e leste do estado do Pará apresentam valores entre 0,51,5 mil FC. No entanto, maior parte do Brasil o número de registros é superior a 100 FC. Este padrão verificado nos totais e nas médias anuais está fortemente associado a três aspectos: i) desmatamento (estados da região Norte), i) expansão dos consórcios agrícolas (ao longo da região Centro-Norte do Brasil), e iii) alterações no clima (região Centro-Oeste). No aspecto do desmatamento na Amazônia (estados do Acre, Pará, Roraima e Rondônia) são resultantes da exploração ilegal de madeira, mineração e de biodiversidade, além da expansão agrícola, conforme identificado por Caúla et al. (2015), Silva Júnior et al. (2019) e Oliveira et al. (2021). 
Em relação à alta incidência de FC situados na região centro-leste está associada às áreas de desmatamento e erosão para a expansão do consórcio agrícola MATOPIBA (composto pelos estados do Maranhão, Tocantins, Piauí e Bahia) - (Santos et al., 2014; Assis et al., 2021), principalmente no estado do Maranhão conforme constatado por Mataveli et al. (2017), Bezerra et al. (2018), Silva Junior et al. (2019) e Assis et al. (2021). Esta mudança no uso e ocupação da terra resultante da ação do desmatamento contribui para a exposição da vegetação e do solo (Correia Filho et al., 2018), e, assim aumenta a probabilidade de ocorrência de incêndios conforme verificado por Oliveira et al. (2021). Sobre os estados de Alagoas, São Paulo (região noroeste), e Minas Gerais estão relacionados a áreas com plantio de cana-de-açúcar, e tal cultivo utiliza o fogo para a colheita (Fernandes \& Correia Filho, 2014; Mataveli et al., 2017; Girardi, 2019; Oliveira-Júnior et al., 2020b; Marinho et al., 2021; Silva et al., 2021).

Figura 3 - Total anual de FC no Brasil nos anos de maior ocorrência: (a) 2015, (b) 2017, (c) 2019 e (d) 2020.

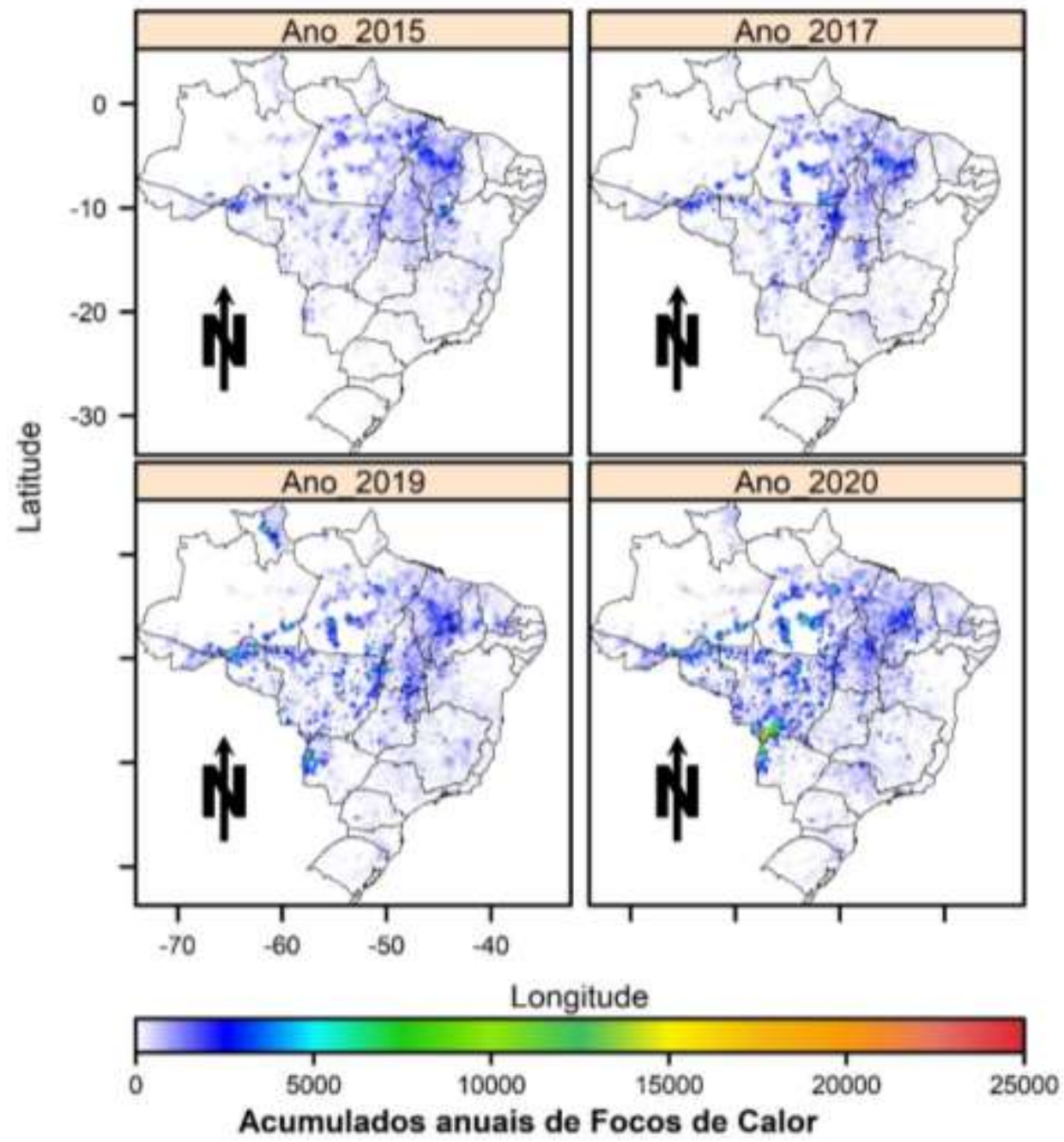

Fonte: BDQUEIMADAS-CPTEC/INPE (2021). Mapa elaborado pelos autores.

Em relação aos estados do Mato Grosso e Mato Grosso do Sul, a alta incidência também está relacionada a expansão da produção agrícola conforme verificado por Oliveira-Júnior et al. (2020b), Guerra et al. (2020). A expansão agrícola associada às alterações no clima (Oliveira-Júnior et al., 2020a) resultaram em secas no bioma Cerrado (Santos et al., 2014; Souza et al., 2020a; Souza et al., 2020b), Amazônico (Alencar et al., 2015; Fonseca et al., 2019), e no Pantanal (Marengo et al., 2021). De acordo com a variabilidade anual de FC, as regiões com altas concentrações apresentam similaridade ao longo dos 
anos, com intensificação no número de registros, após o ano de 2015, sendo ano caracterizado pela atuação de Super El Nino (Clemente et al., 2017). Esse período de alta incidência de FC é relacionado à ocorrência de estiagem prolongada e, assim subsidia a ocorrência desses eventos (Correia Filho et al., 2019; Marengo et al., 2021).

Desta maneira, avaliou-se quatro anos de maior número de registros (Figura 3): (a) 2015, (b) 2017, (c) 2019 e (d) 2020. Os Estados com maior incidência de FC foram Pará, Maranhão, Mato Grosso, Mato Grosso do Sul e Tocantins, que variam entre 2,5-25 mil registros. Esse padrão crescente no número de FC é resultante das ações antrópicas como o desmatamento para o extensivo uso e ocupação do solo para agricultura (Assis et al., 2021). O desmatamento é uma prática comum no processo de transformação de florestas em terras para atividades agropecuárias (Dias et al., 2016; Oliveira et al., 2021; Oliveira-Júnior et al., 2021), sendo verificado em diversas regiões na AS (Fonseca et al., 2017; Diniz et al., 2019; Flores et al., 2021). Este processo de conversão ocorre de duas formas: (i) os agricultores desmatam a floresta, cultivam a terra, deixando-a se restaurar ao estado natural de floresta; e (ii) realizado com a intenção de se estabelecer permanentemente atividades agropecuárias (Assis et al., 2021; Oliveira et al., 2021).

Além dos fatores antrópicos, a atuação do ENOS também condiciona a ocorrência de FC ao longo do Brasil (Santos et al., 2014). Entre os anos de 2014/2015, a atuação do El Niño que persistiu até meados de 2016, ao qual causou enormes prejuízos em diversas regiões do globo com o surgimento de longo período de estiagem, inclusive incêndios no norte da Amazônia conforme detectados por Fonseca et al. (2017), Fonseca et al. (2019) e Diniz et al. (2019). A ocorrência desse El Niño resultou uma forte irregularidade no regime pluviométrico nas regiões Norte e Nordeste do Brasil, principalmente no interior da região Nordeste, devido ao deslocamento da célula de Walker e mudança do seu ramo descendente (Reboita et al., 2010). Essas alterações no padrão chuvoso associados a condições mais atmosféricas de secura oferecem condições favoráveis para o aumento de FC naturais no Brasil, assim como também facilita a propagação de incêndios por indução humana (Vasconcelos et al., 2015; Pereira \& Silva, 2016).

Como consequência a atuação desse El Niño (Figura 3a), constatou-se maiores registros de FC com 10-25 mil no Brasil, destaque na parte norte, principalmente sobre os estados do Tocantins, Maranhão e Piauí (Silva Junior et al., 2019). Houve similaridade entre os anos de 2017, 2019 e 2020 com o ano de 2015, com maiores registros entre 10-25 mil FC. Destaca-se o aumento significativo no número de FC no Mato Grosso do Sul, situado no Bioma Pantanal, com valores entre 10-25 mil FC, motivado pelo longo período de estiagem resultante da escassez de chuvas conforme constatado por Marengo et al. (2021), que resultou em grandes incêndios no período (Flores et al., 2021). Os incêndios florestais pan-tropicais aumentarão a vulnerabilidade das florestas a medida que estas forem danificadas e menos resistentes ao fogo cobrirem a paisagem e ocorrerem episodicamente mais severos durante os eventos de El Niño (Cochrane, 2003). Os incêndios na AS (inclusive no Brasil) estão associados às fases ENOS, mas a ignição também está ligada ao uso da terra e atividades antrópicas, como pastagem e manejo de culturas (Assis et al., 2021; Marinho et al., 2021; Oliveira-Júnior et al., 2021). O IPCC (2014) projeta que a intensificação das mudanças climáticas aumentará a frequência e o tamanho dos incêndios em virtude da recorrência de períodos de secas severas e prolongadas.

O padrão mensal (Figura 4) apresentou características bem distintas, ao qual o período de maior ocorrência de FC está compreendido entre agosto e dezembro, nas regiões Norte, Nordeste e Centro-Oeste, entre 0,2-0,4 mil FC e pico de 1042 FC em agosto e, assim corroborou com os resultados obtidos por Lopes et al. (2017). Esses FC acontecem nessas regiões nos períodos secos devido ao acúmulo de biomassa associados ao clima seco e ventos, entre o inverno e a primavera (Santos et al., 2014). Normalmente estas estações têm como características a ocorrência de veranicos (dry spells), períodos longos de estiagens, baixa umidade do ar e atuação de uma massa de ar seco na região (Reboita et al., 2010; Brito et al., 2016; Oliveira et al., 2021). A detecção dos altos índices de FC corrobora com os resultados encontrados anteriormente por Caúla et al. (2015) quando avaliou FC para o Brasil a partir da análise multivariada (análise de agrupamento - AA), e constataram que período de 
transição entre seco e chuvoso (Rocha et al., 2011; Santos et al., 2014). O período de secura contribui para o estresse da vegetação, tornando-a mais vulnerável à ação do fogo devido à baixa umidade e ausência de chuva nesses meses analisados (Correia Filho et al., 2018).

Figura 4 - Distribuição mensal dos FC no Brasil, durante o período de 1999 e 2020.

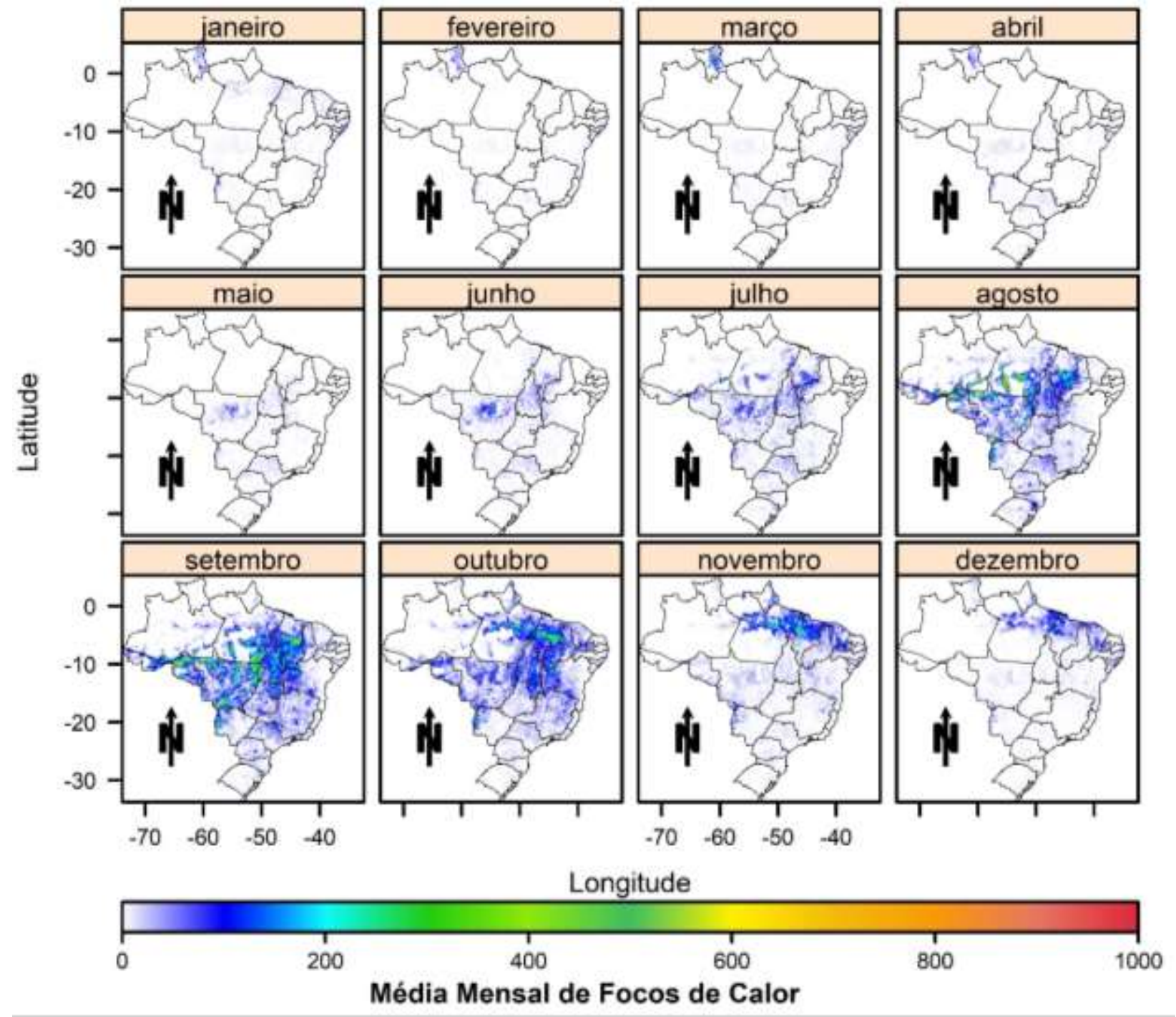

Fonte: BDQUEIMADAS-CPTEC/INPE (2021). Mapa elaborado pelos autores.

O período de menor ocorrência de FC ocorre entre os meses de janeiro e junho (Fig. 3), influenciado pela atuação dos sistemas produtores de chuva no Brasil, como a Zona de Convergência do Atlântico Sul (ZCAS), Zona de Convergência Intertropical (ZCIT) e os sistemas frontais. No caso da ZCAS, atua a partir de outubro até março, sistema responsável pelo período chuvoso das regiões centro-oeste e sudeste que se estende da região norte ao sudeste do Brasil (com eixo diagonal na AS) - (Reboita et al., 2010; Mendes et al., 2019; Climanálise, 2020). Por outro lado, a atuação da ZCIT no extremo norte da AS está intimamente associado a manutenção do regime pluviométrico, inibindo a ocorrência de FC.

As maiores concentrações de FC variou entre 5-47 mil ocorrências, nos limites das regiões Norte, Nordeste e CentroOeste, entre os estados do Bahia, Maranhão, Mato Grosso, Pará, Piauí e Tocantins. Da Silva et al. (2021) apontaram que a proporção de queimadas em áreas recentemente desmatadas foi maior em 2018 e 2019. Em 2019, a contribuição foi de 41\%, seguindo a tendência de aumento do desmatamento no Acre (INPE, 2020). Os autores constatam que as queimadas com áreas 
maiores que 10 hectares (ha) foram maiores em 2019 do que nos anos de 2017 e 2018, representando $32 \%$ da área total mapeada.

Os resultados obtidos corroboram com Fernandes et al. (2019) que no Brasil, o Sistema de Alerta de Desmatamento (SAD) identificou que, em março de 2018, foram desmatados $287 \mathrm{~km}^{2}$ (28.700 ha) na Amazônia Legal. A fração de desmatamento entre 1 e 10 ha foi de $18 \%$ do total detectado (4.400 ha). Considerando somente os alertas a partir de 10 hectares, houve aumento de $249 \%$ em relação a março de 2017, quando o desmatamento somou $71 \mathrm{~km}^{2}$ (7.100 ha). Em março de 2018, o desmatamento ocorreu em Mato Grosso (40\%), Roraima (21\%), Amazonas (14\%) e Rondônia (7\%).

\section{Considerações Finais}

Os resultados obtidos neste estudo diagnóstico apontam que os meses de agosto, setembro e outubro são os mais propensos a ocorrência de FC no Brasil. Em relação aos totais e médios anuais há uma variação estapacial entre 2,5-50 mil FC e 0,5-1,5 mil FC, respectivamente. As maiores concentrações de FC estão situadas no centro-norte do Brasil, principalmente entre os estados do consórcio agrícola MATOPIBA, motivados pela expansão agrícola. Os anos com maiores números de FC coincidiram com o aumento do desmatamento, associado à ocorrência das fases do ENOS, sendo constatados nos últimos anos, ocasionados pelos longos períodos de estiagem causados pela escassez de chuvas, principalmente no Mato Grosso do Sul, no bioma Pantanal.

\section{Agradecimentos}

O primeiro e quinto autores agradecem ao Fundo de Amparo à Pesquisa do Estado de Alagoas (FAPEAL) pela concessão de Bolsa de Iniciação Científica (PIBIC) como colaboradora e bolsista, respectivamente. O segundo autor agradece ao Conselho Nacional para Pesquisa e Desenvolvimento Tecnológico (CNPq) pela bolsa Pós-Doutorado Júnior de $\mathrm{n}^{\circ}$ 161023/2019-3. O terceiro autor agradece ao CNPq pela sua bolsa de Produtividade de Pesquisa (PQ) de ${ }^{\circ} 309681 / 2019-7$.

\section{Referências}

Alencar, A. A., Brando, P. M., Asner, G. P., \& Putz, F. E. (2015). Landscape fragmentation, severe drought, and the new Amazon Forest fire regime. Ecological applications. 25 (6), 1493-1505. https://doi.org/10.1890/14-1528.1

Alvares, C. A., Stape, J. L., Sentelhas, P. C., Gonçalves, J. D. M., \& Sparovek, G. (2013). Köppen's climate classification map for Brazil. Meteorologische Zeitschrift. 22 (6), 711-728.

Assis, T. O., Escada, M. I. S., \& Amaral, S. (2021). Effects of Deforestation over the Cerrado Landscape: A Study in the Bahia Frontier. Land, 10 (4), 352.

Brito, T.T.; Oliveira-Júnior, J.F.; Lyra, G.B.; Góis, G.; Zeri, M. (2016). Multivariate analysis applied to monthly rainfall over Rio de Janeiro state, Brazil. Meteorology and Atmospheric Physics (Print), 129 (5), 469-478.

Caúla, R. H., Oliveira-Júnior, J.F., Lyra, G.B., Delgado, R.C., \& Heilbron Filho, P.F.L. (2015). Overview of fire foci causes and locations in Brazil based on meteorological satellite data from 1998 to 2011. Environmental Earth Sciences (Print), 74, 1497-1508. https://doi.org/10.1007/s12665-015-4142-z.

Climanálise. (2021). Boletins Mensais Climatológicos. http://climanalise.cptec. inpe.br/ rclimanl/boletim/

Cochrane, M. A. (2003). Fire science for rainforests. Nature, 421, 913-919.

Clemente, S. S., Oliveira Júnior, J. F., \& Louzada, M. A. P. (2017). Focos de Calor na Mata Atlântica do Estado do Rio de Janeiro. Revista Brasileira de Meteorologia, 32, 669-677.

Correia Filho, W. L. F., dos Santos, T. V., Diogo, A. M., \& de Amorim, R. F. C. (2018). Diagnóstico da Precipitação e EVI em Dois Eventos de Seca no Nordeste do Brasil. Revista do Departamento de Geografia, 35, 102-112.

Correia Filho, W. L. F., De Oliveira-Júnior, J. F., De Barros Santiago, D., De Bodas Terassi, P. M., Teodoro, P. E., De Gois, G., Blanco, C. J. C., P. H. A., Costa, M. S., Gomes, H. B., \& Dos Santos, P. J. (2019). Rainfall variability in the Brazilian northeast biomes and their interactions with meteorological systems and ENSO via CHELSA product. Big Earth Data, 3 (4), 315-337. https://doi.org/10.1080/20964471.2019.1692298 
Dias, L. C., Pimenta, F. M., Santos, A. B., Costa, M. H., \& Ladle, R. J. (2016). Patterns of land use, extensification, and intensification of Brazilian agriculture. Global change biology. 22 (8), 2887-2903. https://doi.org/10.1111/gcb.13314

Diniz, F. R. et al. (2019). The impact of el niño on fire outbreaks and human thermal discomfort in Brazil in the period between summer of $2014 / 2015$ until the autumn of 2016. Anuário do Instituto de Geociencias, 192-201.

Fernandes, T., Hacon, S. S., Novais, J. W. Z., Souza, I. P., \& Fernandes, T. (2018) Detecção e análise de focos de calor no município de Parauapebas-PA, Brasil por meio da aplicação de geotecnologia. Enciclopédia Biosfera, 15 (28), 398-412. doi: http://dx.doi.org/10.18677/EnciBio_2018B34

Fernandes, R.C., \& Correia Filho, W.L.F. (2013). Espacialização temporal dos focos de queimadas e de poluentes atmosféricos $\left(\mathrm{CO}\right.$, $\mathrm{CH}_{4}$, $\mathrm{NO}_{2}$, $\left.\mathrm{N}_{2} \mathrm{O}\right)$ em Alagoas. Ciência e Natura, 35 (2), 287-294.

Flores, B. M. et al. (2021). Tropical riparian forests in danger from large savanna wildfires. Journal of Applied Ecology, 58 (2), $419-430$.

Fonseca, M. G. et al. (2017). Climatic and anthropogenic drivers of northern Amazon fires during the 2015-2016 El Niño event. Ecological applications, 27 (8), 2514-2527.

Fonseca, M. G. et al. (2019). Effects of climate and land-use change scenarios on fire probability during the 21st century in the Brazilian Amazon. Global change biology, 25 (9), 2931-2946.

Girardi, E. P. (2019). Agronegócio sucroenergético e desenvolvimento no Brasil. Confins. Revue franco-brésilienne de géographie/Revista franco-brasilera de geografia, 40. https://doi.org/10.4000/confins.19517

Guerra, A. et al. (2020). The importance of Legal Reserves for protecting the Pantanal biome and preventing agricultural losses. Journal of environmental management, 260, 110128.

IBGE - Instituto Brasileiro de Geografia e Estatística (2021). IBGE apresenta nova área territorial brasileira: 8.515.767,049 km 2. Rio de Janeiro, 2012a. https://agenciadenoticias.ibge.gov.br/agencia-sala-de-imprensa/2013-agencia-de-noticias/releases/14318-asi-ibge-apresenta-nova-area-territorialbrasileira8515767049-km

INPE - Instituto Nacional De Pesquisas Espaciais. (2020). Project PRODES - Monitoring of the Brazilian Amazon Forest by Satellite. http://www.obt.inpe.br/OBT/assuntos/programas/amazonia/prodes

INPE - Instituto Nacional De Pesquisas Espaciais. (2021). Dados pontuais de focos ativos de calor - Sistema de Detecção de Desmatamento em Tempo RealDETER. http://www.obt.inpe.br/OBT/assuntos/programas/amazonia/deter/deter

IPCC - Intergovernmental Panel on Climate Change (2014). Summary for policymakers. In: Climate Change 2014: Impacts, Adaptation, and Vulnerability. Part A: Global and Sectoral Aspects. Contribution of Working Group II to the Fifth Assessment Report of the Intergovernmental Panel on Climate Change [Field, C.B., V.R. Barros, D.J. Dokken, K.J. Mach, M.D. Mastrandrea, T.E. Bilir, M. Chatterjee, K.L. Ebi, Y.O. Estrada, R.C. Genova, B. Girma, E.S. Kissel, A.N. Levy, S. MacCracken, P.R. Mastrandrea, and L.L.White (eds.)]. Cambridge University Press, Cambridge, United Kingdom and New York, 1-32.

Jarvis A., Reuter, H. I., Nelson, A., Guevara, E. (2008). Hole-filled seamless SRTM data V4. International Centre for Tropical Agriculture (CIAT) http://srtm.csi.cgiar.org

Landau, E. C., Moura, L. Configuração territorial do Brasil: divisão política, biomas, características demográficas e socioeconômicas. Embrapa Milho e Sorgo-Capítulo em livro científico (ALICE), 2020.

Lopes, A. C. L. et al. (2017). Análise da distribuição de focos de calor no município de Novo Progresso, Pará. Revista Verde de Agroecologia e Desenvolvimento Sustentável, 12 (2), 298-303.

Marengo, J. A. et al. (2021). Extreme Drought in the Brazilian Pantanal in 2019-2020: Characterization, Causes, and Impacts. Frontiers in Water, 3, 639204.

Marinho, A. A. R. et al. (2021). Temporal record and spatial distribution of fire foci in State of Minas Gerais, Brazil. Journal of Environmental Management, 280, 111707. https://doi.org/10.1016/j.jenvman.2020.111707

Mataveli, G. A. V. et al. (2017). Análise das queimadas e da precipitação em áreas de Cerrado do Maranhão a partir de dados do sensor MODIS e do satélite TRMM para o período 2002-2015. Boletim Paulista de Geografia, 96 (11-30).

Mendes, M. C. D., Da Silva Aragão, M. R, \& De Fatima Correia, M. (2019). Bloqueios Atmosféricos sobre os Oceanos Pacífico Sudeste e Atlântico Sul: Características Sinótico-Dinâmicas e Termodinâmicas. Anuário do Instituto de Geociências, 42 (2), 309-324.

Nascimento Silva, P., Escobar, G. C. J., \& Reboita, M. S. (2020). Eventos extremos de precipitação no Estado de Minas Gerais associados com a ocorrência de episódios de Zona de Convergência do Atlântico Sul. Revista Brasileira de Geografia Física, 13 (3), $1013-1023$.

Oliveira, U. et al. (2021). Modeling fuel loads dynamics and fire spread probability in the Brazilian Cerrado. Forest Ecology and Management, 482 , 118889. https://doi.org/10.1016/j.foreco.2020.118889

Oliveira-Júnior, J. F. et al. (2020a). Fire foci related to rainfall and biomes of the state of Mato Grosso do Sul, Brazil. Agricultural and Forest Meteorology, 282,107861 .

Oliveira-Júnior, J. F. et al. (2020b). Fire foci dynamics and their relationship with socioenvironmental factors and meteorological systems in the state of Alagoas, Northeast Brazil. Environmental Monitoring and Assessment, 192 (10), 1-26.

Oliveira, B. C. C. et al. (2021). Spatiotemporal variation of dry spells in the State of Rio de Janeiro: geospatialization and multivariate analysis. Atmospheric Research, 1, 105612.

Pereira, J.A.V., \& Silva, J. B. (2016). Detecção de Focos de Calor no Estado da Paraíba: Um Estudo Sobre As Queimadas. Revista Geográfica Acadêmica, 10 (1), 5-16. 
Research, Society and Development, v. 10, n. 7, e3731071612, 2021

(CC BY 4.0) | ISSN 2525-3409 | DOI: http://dx.doi.org/10.33448/rsd-v10i7.16612

R Development Core Team. (2019). R: A language and environment for statistical computing version 3.6-1. R Foundation for Statistical Computing, Vienna, Austria. http://www.r-project.org, ISBN 3-900051-07-0

Reboita, M. S., Gan, M.A., Rocha, R.P., \& Ambrizzi, T. (2010). Regimes de Precipitação na América do Sul: Uma Revisão Bibliográfica. Revista Brasileira de Meteorologia, 25 (2), 185-204.

Rocha, G. F., Ferreira, L. G., Ferreira, N. C., \& Ferreira, M. E. (2011). Detecção de desmatamentos no bioma Cerrado entre 2002 e 2009: padrões, tendências e impactos. Revista Brasileira de Cartografia, 63 (3), 341-349.

Santos, P. R., Pereira, G., \& Rocha, L. C. (2014). Análise da distribuição espacial dos focos de queimadas para o bioma Cerrado (2002-2012). Caderno de Geografia, 24 (1), 133-142.

Silva, S. S., et al. (2021). Burning in southwestern Brazilian Amazonia, 2016-2019. Journal of Environmental Management, $286,112189$.

Silva Júnior, L. A. S. et al. (2019). Fire dynamics in extreme climatic events in western amazon. Environmental Development, $32,100450$.

Souza, J., Martins, P., \& Druciaki, V. (2020a). Uso e cobertura do solo no Cerrado: panorama do período de 1985 a 2018. Élisée - Revista De Geografia Da $U E G, 9(2), \mathrm{e} 922020$.

Souza, A. A. et al. (2020). Dynamics of savanna clearing and land degradation in the newest agricultural frontier in Brazil. GIScience \& Remote Sensing, 57 (7), 965-984.

Silva, E. M. S. et al. (2021). Espaço-Temporalidade dos Focos de Calor na Região Metropolitana de Maceió. Revista Brasileira de Meteorologia, 35, 10291043 .

Urrutia-Jalabert, R., González, M. E., González-Reyes, Á., Lara, A., \& Garreaud, R. (2018). Climate variability and forest fires in central and south-central Chile. Ecosphere, 9(4), e02171.

Xiong, Q., et al. (2020). Fire from policy, human interventions, or biophysical factors? Temporal-spatial patterns of forest fire in southwestern China. Forest Ecology and Management, 474, 118381 .

Vasconcelos, S. S. et al. (2015). Suscetibilidade da vegetação ao fogo no sul do Amazonas sob condições meteorológicas atípicas durante a seca de 2005. Revista Brasileira de Meteorologia, 30 (2), 134-144. 